\title{
Purification of Chlamydia trachomatis by a simple and rapid filtration method
}

\author{
Steven Campbell, ${ }^{*}$ Peter S. Yates, Fiona Waters and Shirley J. Richmond \\ Division of Virology, Department of Pathological Sciences, Stopford Building, University of Manchester, Oxford Road, \\ Manchester M13 9PT, UK
}

(Received 29 October 1990; revised 25 February 1991; accepted 15 March 1991)

\begin{abstract}
A simple method for filter purification of Chlamydia trachomatis from cell culture is described. Crude homogenates of chlamydiae-infected cells were passed through a glass prefilter and a $0.6 \mu \mathrm{m}$ pore diameter polycarbonate filter. The filtrate was then passed through a $0.2 \mu \mathrm{m}$ pore diameter filter on which the chlamydiae were trapped. This filter was then back-washed to collect the organisms. These procedures removed cell debris and soluble protein, and yielded particles with a narrow size distribution. The mean yield of viable chlamydiae purified by filtration was $64 \%$ when the filters were washed at each stage of the process.
\end{abstract}

\section{Introduction}

Chlamydia trachomatis is an intracellular parasite which causes lysis of the host eukaryotic cell. The parasite may therefore be recovered from cultures of infected eukaryotic cells by sampling the growth medium after host cell lysis has occurred. The efficiency of this recovery procedure can be significantly improved by mechanically disrupting the surviving infected host cells at the time of harvesting. When chlamydiae are recovered by either of these procedures, the sample contains fragments of disrupted host cells. In some experimental situations it is necessary to remove host-cell debris. Density gradient centrifugation followed by high-speed sedimentation has been used to purify viable infectious organisms. However, centrifugation does not separate the elementary body form and the larger reticulate body forms (Howard et al., 1974). Bose \& Paul (1982) found that a combination of filtration and centrifugation produced a preparation of chlamydial particles which were $300 \mathrm{~nm}$ in diameter. In addition, others have measured incorporation of radiolabel into chlamydiae by collecting the organisms on $0.2 \mu \mathrm{m}$ pore diameter membrane filters (Allan \& Pearce, 1982). It is also possible to evaluate directly the number of particles trapped on polycarbonate membranes using immunofluorescence microscopy (Knight et al., 1989). A very simple and rapid filtration procedure for purifying viable chlamydiae is now described, which combines removal

Abbreviation: i.f.u., inclusion forming units. of host-cell debris by prefiltration and trapping of the organisms on a membrane filter.

\section{Methods}

Production of purified radiolabelled chlamydiae. The filtration procedure was developed in part by using an isolate of Chlamydia trachomatis (serotype E, strain T181 ; Sompolinsky \& Richmond, 1974) which had been radiolabelled with $\left[{ }^{14} \mathrm{C}\right]$ threonine (Allan \& Pearce, 1982), then purified by density-gradient centrifugation (Howard et al., 1974). Briefly, $2 \times 10^{6}$ inclusion forming units (i.f.u.) of chlamydiae were centrifuged $\left(2400 \mathrm{~g}, 30 \mathrm{~min}, 35^{\circ} \mathrm{C}\right)$ on to a confluent monolayer of McCoy cells within a $75 \mathrm{~cm}^{2}$ flask. The growth medium used was a $1: 1$ mixture of Ham's nutrient mixture F12 and Dulbecco's Modified Eagle Medium (DMEM) which contained 15 mM-HEPES buffer (Sigma). It was supplemented with $10 \%(\mathrm{v} / \mathrm{v})$ foetal calf serum, $2 \mathrm{mM}$-glutamine and $100 \mu \mathrm{g}$ streptomycin $\mathrm{ml}^{-1}$. One day later the growth medium was replaced with $11.5 \mathrm{ml}$ threonine-free Minimal Essential Medium (Select-Amine, Gibco) supplemented with $0.5 \%$ foetal calf serum, $4 \mu \mathrm{g}$ cycloheximide $\mathrm{ml}^{-1}$ and $25 \mu \mathrm{Ci}(925 \mathrm{kBq})\left[{ }^{14} \mathrm{C}\right]$ threonine $(228 \mu \mathrm{Ci}$ $\mathrm{mmol}^{-1}, 8.44 \mathrm{MBq} \mathrm{mmol}{ }^{-1}$; Amersham), and the culture was incubated for a further $48 \mathrm{~h}$. A control flask of uninfected cells was labelled in the same way and subjected to similar purification procedures.

The infected cells were mechanically disrupted by shaking with glass beads $(1.5-2 \mathrm{~mm})$. The homogenate was centrifuged at $5000 \mathrm{~g}$ for $10 \mathrm{~min}$ and the supernatant was saved. The pelleted material was resuspended in PBS, centrifuged through a $7 \%(w / v)$ layer of sucrose and a second supernatant fraction was collected (Bose \& Paul, 1982). Both supernatant fractions were then combined and centrifuged at $50000 \mathrm{~g}$ for $60 \mathrm{~min}$. The resulting pellet was resuspended in $0.5 \mathrm{ml}$ PBS (Dulbecco's PBS without divalent cations, $\mathrm{pH} 7 \cdot 2$ ), layered on to a $32-$ $50 \%$ gradient of Urografin 370 (Schering) and centrifuged at $25000 \mathrm{~g}$ for $120 \mathrm{~min}$.

In initial experiments, the amount of radiolabel that appeared in sequential gradient fractions of centrifuged preparations from infected 
and uninfected cultures was measured by scintillation counting. The bulk of the radiolabel present in infected (but not uninfected) cultures was present in a fraction with a density of $1.19 \mathrm{~g} \mathrm{ml}^{-1}$ which corresponded to two optically distinct bands that formed within the gradient. These bands were drawn off, pooled and centrifuged at $50000 \mathrm{~g}$ for $60 \mathrm{~min}$. The pellet was resuspended in cell culture medium (equal parts Ham's F12 and DMEM; Sigma) containing 10\% (w/v) sorbitol and stored at $-70^{\circ} \mathrm{C}$. After thawing, the chlamydiae were harvested by centrifugation at $13000 \mathrm{~g}$ and resuspended in tissue culture medium. In order to verify that the radiolabel had become fully incorporated, $100 \mu \mathrm{l}$ of the relevant gradient fractions or bands were added to a glass disc (GFC, Whatman), precipitated with $40 \%$ (w/v) trichloroacetic acid, washed with ethanol and incorporation of radiolabel was then measured by scintillation counting.

Detection of chlamydiae by immunofluorescence. Aliquots of crude infected cell homogenate or chlamydiae purified by density gradient centrifugation or by filtration (see below) were centrifuged at $2400 \mathrm{~g}$ for $30 \mathrm{~min}$ on to scored glass coverslips $(10 \mathrm{~mm}$ diam.) within flatbottomed plastic tubes. The chlamydiae were then fixed in ethanol and stained either directly with a FITC-labelled anti- $C$. trachomatis monoclonal antibody and a rhodamine counterstain (Syva), or indirectly with a mouse monoclonal antibody raised to chlamydial lipopolysaccharide followed by FITC-conjugated goat anti-mouse immunoglobulin (Sigma). Portions of polycarbonate filters were also examined with the same reagents, so that the presence of adherent chlamydiae could be detected. The filter housings were disassembled immediately after use and transferred to a Petri dish containing ethanol. After fixation, the pieces of filter were transferred to a microscope slide, washed with PBS and stained as above. All specimens were mounted in non-fade mountant containing $p$ phenylenediamine (Johnson et al., 1981) and examined and photographed with epi-illumination using $\times 63$ and $\times 100$ objectives. Specimens on coverslips were also examined with phase-contrast optics.

Development of the filtration procedure. Polycarbonate pore filters (Nuclepore, Costar UK) have been shown to be suitable for the filtration of chlamydiae (Bose \& Paul, 1982). However, our preliminary experiments with crude infected cell homogenates demonstrated that it was necessary to use a glass prefilter within the same housing (Swinlock, Costar UK) in order to prevent clogging of the polycarbonate filters. Qualitative immunofluorescence of the filtrates demonstrated that some chlamydiae were retained on the glass prefilters. A thin prefilter $(0.356 \mathrm{~mm})$ available from the same manufacturer (D49, Nuclepore, Costar UK) was therefore used in order to minimize loss of chlamydiae.

Polycarbonate filters $(25 \mathrm{~mm}$ diameter) of 0.4 or $0.6 \mu \mathrm{m}$ pore diameter were then tested in combination with the glass prefilter for their ability to permit the passage of centrifugally purified radiolabelled organisms. The filtrates from the 0.4 or $0.6 \mu \mathrm{m}$ filtrations were passed through a polycarbonate filter of a smaller pore diameter $(0.2 \mu \mathrm{m})$ which trapped the chlamydial elementary bodies (Allan \& Pearce, 1982; Knight et al., 1989). After chlamydial trapping, the $0.2 \mu \mathrm{m}$ filter was washed through with tissue culture medium to remove small debris. The chlamydiae trapped on the $0.2 \mu \mathrm{m}$ filter were collected by back-washing with culture medium, with or without $10 \%$ $(w / v)$ sorbitol. Some filter assemblies have filter supports and adaptors which permit a syringe to be fitted in the reverse direction and so allow back-washing (e.g. $47 \mathrm{~mm}$ Swinlok, Costar, UK). If such an adaptor is not available, one may be easily constructed by cutting off the plastic ends of two disposable syringe needles and forcing one of these pieces into each end of a small piece of silicone rubber tubing, so that there is a syringe-compatible fitting at both ends.
The separation of soluble cellular components and culture medium from particles during the second filtration step was monitored by examination of this filtrate and subsequent washings. Washing through with PBS was continued beyond the point at which the phenol red, present in the tissue culture medium, was no longer detectable by eye. The amounts of soluble material present in filtrate and 15 consecutive $2 \mathrm{ml}$ aliquots of the washings was then determined by measuring $A_{\mathbf{2 8 0}}$. The presence of proteins in the washings and in the final chlamydial suspension was measured colorimetrically with a commercial protein assay kit (BCA, Pierce) used at $56^{\circ} \mathrm{C}$ for enhanced sensitivity. Chlamydiae were removed from aliquots of the final suspension by centrifugation at $13000 \mathrm{~g}$ for $15 \mathrm{~min}$ in small conical vials (Sarstedt), and the protein assay repeated.

Efficiency of recovery of viable chlamydiae by the filtration method. Flasks $\left(25 \mathrm{~cm}^{2}\right)$ of confluent McCoy cells were infected with $2 \times 10^{6}$ i.f.u. of chlamydiae and cultured for $72 \mathrm{~h}$ in $5 \mathrm{ml}$ of growth medium. The infected cells were disrupted and $0.5 \mathrm{ml}$ of the homogenate was retained. The remaining $4.5 \mathrm{ml}$ was filtered through a filter housing containing a glass prefilter (D49, Nuclepore) and a $0.6 \mu \mathrm{m}$ pore diameter polycarbonate filter ( 25 or $47 \mathrm{~mm}$ diameter). In later experiments the efficiency of the whole procedure was increased by washing the filters with $10 \mathrm{ml}$ PBS to remove chlamydiae. Chlamydiae in the filtrate were then trapped on a $0.2 \mu \mathrm{m}$ pore polycarbonate filter and washed with $10 \mathrm{ml}$ of unsupplemented growth medium or PBS. This filter was then back-washed with $5 \mathrm{ml}$ of medium for $25 \mathrm{~mm}$ diameter filters or $10 \mathrm{ml}$ for the $47 \mathrm{~mm}$ diameter filter to collect chlamydiae. In later experiments with $47 \mathrm{~mm}$ diameter filters, the back-wash volume was increased to between 15 and $20 \mathrm{ml}$. Tenfold serial dilutions of the unfiltered homogenate and the filter-purified chlamydial suspension were inoculated, in duplicate, on to coverslips of confluent McCoy cells. The numbers of i.f.u. present in the crude homogenate and filter-purified suspension were determined by immunofluorescent staining of the monolayers after $48 \mathrm{~h}$ incubation.

Size analysis of isolated particles by scanning electron microscopy. The size and shape distribution of purified particles isolated by densitygradient centrifugation or filtration were assessed by scanning electron microscopy and semi-automatic image analysis. The purified particles were centrifuged on to glass coverslips as indicated above, fixed wtih $2.5 \%(\mathrm{v} / \mathrm{v})$ glutaraldehyde in unsupplemented growth medium for at least $1 \mathrm{~h}$, post-fixed in $1 \%(\mathrm{w} / \mathrm{v})$ aqueous osmium tetroxide, dehydrated, critical-point freeze-dried from liquid carbon dioxide and sputter-coated with approximately $5 \mathrm{~nm}$ of gold. The specimens were then examined with a scanning electron microscope (SEM S360, Cambridge Instruments) at a nominal magnification of $\times 28000$.

The secondary electron image was recorded directly from the frame store of the microscope onto U-matic video tape for image analysis. The shapes of all particles were analysed semi-automatically from the video image using a microcomputer-based measuring system (Measure Mouse, Analytical Measuring Systems). The longest dimension (i.e. longest distance separating two points on the cell perimeter), breadth (perpendicular to the longest dimension), axial ratio (breadth/length) and circularity $\left(4 \times\right.$ area/perimeter $\left.{ }^{2}\right)$ of objects from each specimen were then analysed. The $95 \%$ confidence intervals of the means of each variable were calculated for both methods of chlamydial isolation with Minitab Release 7 (Minitab Inc.). The differences in the means of each variable were analysed by two-tailed $t$-tests.

\section{Results}

Preliminary experiments were carried out to assess the effect of pore diameter on the passage of chlamydiae through polycarbonate filters. Centrifugally purified chlamydiae were passed through polycarbonate filters 

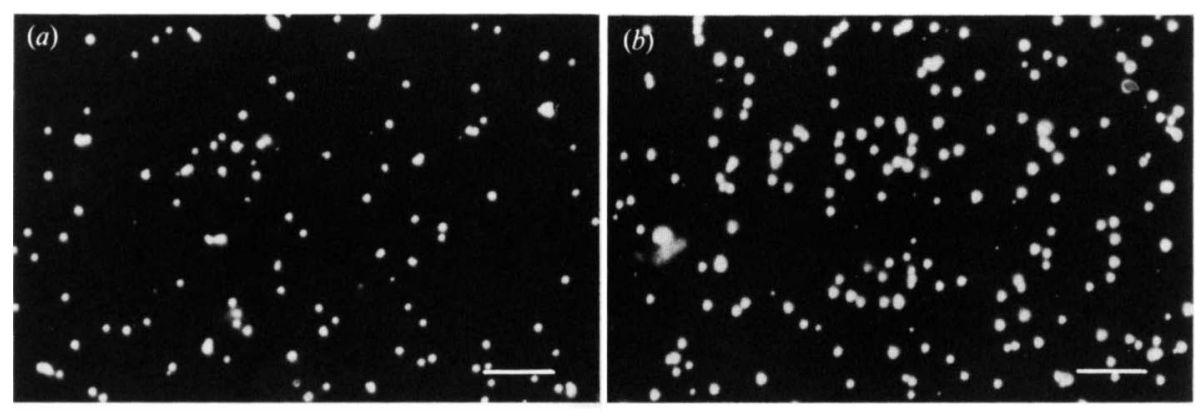

Fig. 1. Chlamydiae purified by centrifugation on Urografin gradients were passed through (a) $0.4 \mu \mathrm{m}$ or (b) $0.6 \mu \mathrm{m}$ pore diameter polycarbonate pore filters. The chlamydiae were then centrifuged on to coverslips after filtration and visualized by indirect immunofluorescence. The filtrate from the $0.6 \mu \mathrm{m}$ filter contained many more fluorescent particles. Bar, $10 \mu \mathrm{m}$.
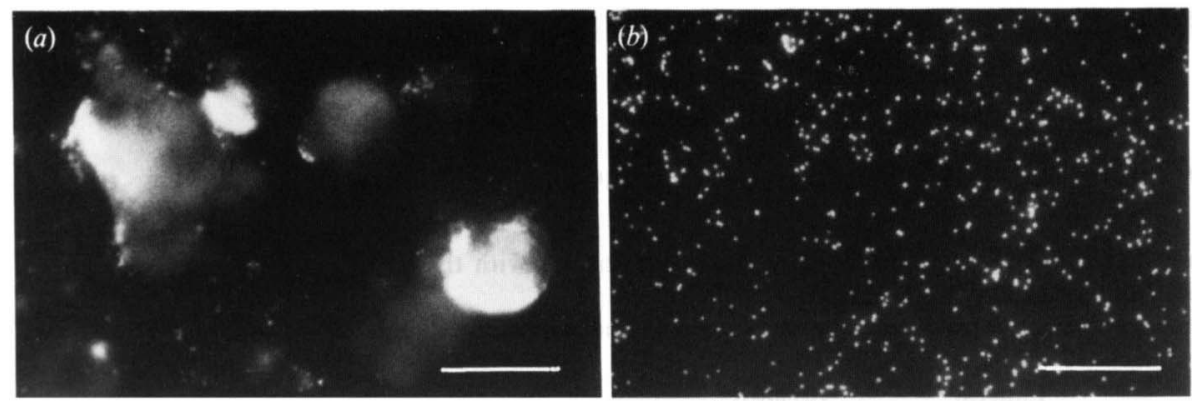

Fig. 2. A cell homogenate stained with a FITC-conjugated anti-chlamydial antibody and a rhodamine counterstain, before and after filtration through a glass prefilter and a $0.6 \mu \mathrm{m}$ pore diameter polycarbonate filter. (a) A crude homogenate of chlamydiae-infected cells before filtration showing large pieces of cell debris identified with the rhodamine counterstain. (b) The same homogenate after filter purification contained no large pieces of cell debris. Bar, $10 \mu \mathrm{m}$.

with a pore diameter of either 0.4 or $0.6 \mu \mathrm{m}$ and the filtrates were examined by indirect immunofluorescence. More chlamydial particles were observed after passage through the $0.6 \mu \mathrm{m}$ filter (Fig. 1). Scintillation counting indicated that approximately $11 \%$ of the centrifugally purified radiolabelled organisms were retained by the $0.6 \mu \mathrm{m}$ filter when the filter was washed with PBS. In two experiments, an average of four times greater retention occurred on the $0.4 \mu \mathrm{m}$ filters.

In order to purify large numbers of chlamydiae from infected cell homogenates, $0.6 \mu \mathrm{m}$ filters were used in combination with glass prefilters (see Methods). Large fragments of cellular debris in the crude cellular homogenates were removed by the combined effect of the glass prefilter and the $0.6 \mu \mathrm{m}$ polycarbonate filter (Fig. 2). Prior to the first filtration step, the homogenate was very turbid. After this step, the particle suspension was visibly clarified. Clarification of the chlamydial suspension should therefore be used as a visual check on whether the first stage of the procedure has worked satisfactorily.

In the second filtration step, the chlamydiae were trapped on the $0.2 \mu \mathrm{m}$ filter. The filter was then washed with PBS to remove soluble components of the homogenate. The $47 \mathrm{~mm}$ diameter filter housings required a through-wash of $16 \mathrm{ml}$ before the $A_{280}$ of the filtrate fell to undetectable levels. At this stage there was no detectable protein (i.e. less than $50 \mu \mathrm{g} \mathrm{ml}^{-1}$ ) present in the filtrate. The filter was then back-washed with PBS to remove the trapped chlamydiae. No contaminating protein was detected in the final chlamydial suspension or in the supernatant fraction after chlamydiae were removed by centrifugation. Indeed there was no detectable protein at all in the chlamydial suspension (the total mass of viable chlamydial elementary bodies per millilitre of suspension would be in the order of $1 \mu \mathrm{g}$ ). Immunofluorescence microscopy demonstrated that chlamydiae were retained on the $0.2 \mu \mathrm{m}$ filter after washing. No attempt was made, however, to quantify the numbers of particles retained or to measure their size distribution.

Scanning electron microscopy of chlamydiae purified from cell homogenates by either density-gradient centrifugation or filtration revealed that both sets of particles were qualitatively similar in shape, being approximately spherical with a smooth external surface (Fig. 3). Size and shape analysis of the scanning electron micrography images stored on video tape (Table 1) revealed that particles purified by centrifugation were more heterogeneous in size and were on average slightly larger and slightly less circular in projected outline than those isolated by filtration. These results are consistent with the fact that centrifugation selects particles on the basis of density whereas filtration selects on the basis of size. The absence of cellular debris less than $0 \cdot 2 \mu \mathrm{m}$ diameter in the filter-purified suspensions was a further indication that contaminating material was removed from the $0 \cdot 2 \mu \mathrm{m}$ filter by washing. 

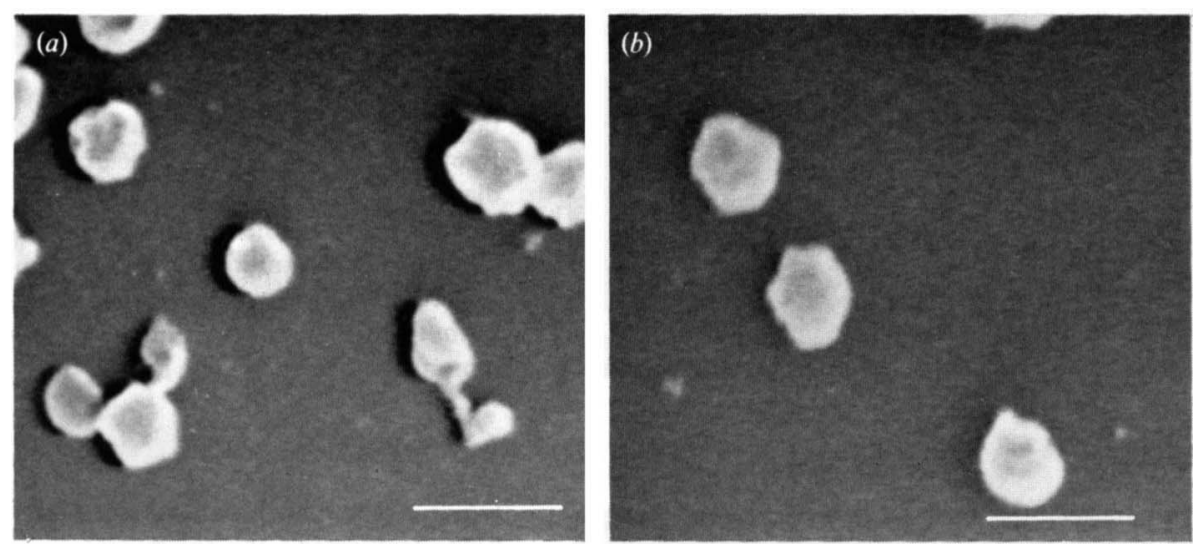

Fig. 3. Frames from video tape of scanning electron microscope images showing chlamydiae purified by $(a)$ density-gradient centrifugation through Urografin or $(b)$ by filtration. Bar, $0.4 \mu \mathrm{m}$.

Table 1. Shape and size analysis of particles identified by scanning electron microscopy

See Methods for definition of the parameters. The results are means, with the $95 \%$ confidence limits of the mean in parentheses.

\begin{tabular}{lcccc}
\hline & $\begin{array}{c}\text { Longest } \\
\text { dimension }(\mu \mathrm{m})\end{array}$ & $\begin{array}{c}\text { Breadth } \\
(\mu \mathrm{m})\end{array}$ & Axial ratio & Circularity \\
\hline Filter-purified & 0.34 & 0.28 & 0.84 & 0.88 \\
particles $(n=192)$ & $(0.32-0.36)$ & $(0.27-0.30)$ & $(0.83-0.66)$ & $(0.86-0.81)$ \\
Centrifugally-purified & 0.47 & 0.39 & 0.83 & 0.83 \\
particles $(n=96)$ & $(0.42-0.53)$ & $(0.34-0.43)$ & $(0.81-0.89)$ & $(0.81-0.86)$ \\
$P^{*}$ & $<0.001$ & $<0.001$ & 0.5 & 0.0025 \\
\hline \hline
\end{tabular}

* Probability that sample means of each variable are not significantly different.

The yields of viable elementary bodies recovered by filtration of the homogenized contents of individually infected $25 \mathrm{~cm}^{2}$ flasks using a $25 \mathrm{~mm}$ diameter filter set (glass prefilter, and 0.6 and $0.2 \mu \mathrm{m}$ polycarbonate filters) were 47 and $63 \%$ in the first two experiments. However, in two subsequent experiments these small-diameter filters clogged. Small filters such as these should therefore be used only on small (frozen) aliquots of crude chlamydial homogenates. When the filtration was repeated with $47 \mathrm{~mm}$ diameter filter sets, a mean $30 \%$ recovery rate was obtained from the first five experiments, which yielded 15.9, 15.6, 17.2, 58.3 and $42.8 \%$ individual recovery rates. The proportion of the total incorporated radiolabel recovered by filtration of crude infected cell homogenate in one pair of experiments was $17.7 \%$, compared to $11 \%$ recovered by density-gradient centrifugation.

In order to improve the yield and reliability of the method, a second set of experiments was undertaken in which the first set of filters (prefilter plus $0.6 \mu \mathrm{m}$ pore diameter polycarbonate filter) were washed so that chlamydiae trapped on the filter could be recovered. The addition of this step resulted in a higher yield of viable organisms. Although the yield continued to have some unexplained variability, a mean of $64 \%$ was recorded over seven experiments, in which the individual percentage recovery rates of viable organisms were $76 \cdot 5,96 \cdot 3$, $71 \cdot 0,66 \cdot 2,82 \cdot 7,10 \cdot 9$ and $47 \cdot 6 \%$. The average total yield of viable organisms obtained by this procedure from one $25 \mathrm{~cm}^{2}$ flask was $1.8 \times 10^{8}$ i.f.u.

\section{Discussion}

The filtration method described here is very convenient for small- to medium-scale purification of chlamydiae from in vitro cultures. One of the major advantages of this technique over centrifugal purification is its rapidity, for the organisms may be isolated within a few minutes. If maximum yield is required as well as a strict criterion of purity, as when radiolabelling, an additional short centrifugation step may be used to pellet the chlamydiae. Where a greater stringency in the range of particle size is required, it may be better to use a $0.4 \mu \mathrm{m}$ pore diameter 
polycarbonate filter (Bose \& Paul, 1982) rather than the larger $0.6 \mu \mathrm{m}$ pore diameter filter preferred in this study. However, in that case a lower yield of organisms would be obtained.

It is not intended that the filtration method described here should be treated as a replacement for densitygradient centrifugation, which is more suitable for largescale purification of chlamydiae. At present our method is restricted by the maximum filter area that is practical to use; for example, a $47 \mathrm{~mm}$ diameter filter can be used to isolate chlamydiae from a single 25 or $75 \mathrm{~cm}^{2}$ tissue culture flask. Larger-scale isolation would require a convenient and sterilizable tangential flow system, which is not readily available. Yolk sac cultures of chlamydiae also require centrifugal purification, as the crude supernatant fractions of this material block the filters. This rapid filtration method has proved to be particularly useful in cell culture experiments which require viable chlamydiae free of contaminating cell debris.
This work was supported by an MRC project grant.

\section{References}

Allan, I. \& Pearce, J. H. (1982). Radiolabelling of Chlamydia psittaci (strain guinea pig inclusion conjunctivitis) to high specific activity using ${ }^{14} \mathrm{C}$-labelled amino acids. FEMS Microbiology Letters 13, 6973.

Bose, S. K. \& Paul, R. G. (1982). Purification of Chlamydia trachomatis lymphogranuloma venereum elementary bodies and their interaction with HeLa cells. Journal of General Microbiology 128, 1371-1379.

Howard, L., OREnstein, N. S. \& KING, N. W. (1974). Purification on Renografin density gradients of Chlamydia trachomatis grown in the yolk sac of eggs. Applied Microbiology 27, 102-106.

Johnson, G. D. \& Nogueira Araujo, G. M. DE C. (1981). A simple method of reducing the fading of immunofluorescence microscopy. Journal of Immunological Methods 43, 349-350.

K NIGHT, S. T., NeECE, V. R. \& WITT, D. J. (1989). Rapid cultureindependent techniques for quantitation of Chlamydia trachomatis elementary bodies. Journal of Microbiological Methods 10, 255-263.

SompolinsKy, D. \& Richmond, S. J. (1974). Growth of Chlamydia trachomatis in McCoy cells treated with cytochalasin B. Applied Microbiology 28, 912-914. 